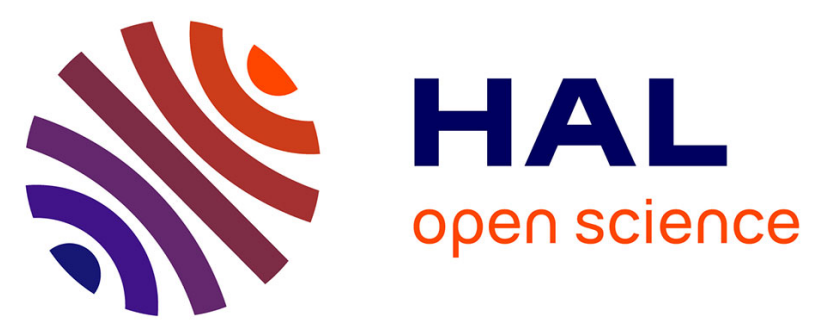

\title{
Comparison of Energetic Macroscopic Representation and structural representation on EV simulation under Simcenter Amesim
}

\author{
Călin Husar, Mihai Grovu, Cristi Irimia, Anatole Desreveaux, Alain \\ Bouscayrol, Matthieu Ponchant, Pacôme Magnin
}

\section{To cite this version:}

Călin Husar, Mihai Grovu, Cristi Irimia, Anatole Desreveaux, Alain Bouscayrol, et al.. Comparison of Energetic Macroscopic Representation and structural representation on EV simulation under Simcenter Amesim. IEEE VPPC'19, Oct 2019, Hanoi, Vietnam. hal-02925439

\section{HAL Id: hal-02925439 \\ https://hal.science/hal-02925439}

Submitted on 29 Aug 2020

HAL is a multi-disciplinary open access archive for the deposit and dissemination of scientific research documents, whether they are published or not. The documents may come from teaching and research institutions in France or abroad, or from public or private research centers.
L'archive ouverte pluridisciplinaire HAL, est destinée au dépôt et à la diffusion de documents scientifiques de niveau recherche, publiés ou non, émanant des établissements d'enseignement et de recherche français ou étrangers, des laboratoires publics ou privés. 


\title{
Comparison of Energetic Macroscopic Representation and structural representation on EV simulation under Simcenter Amesim
}

\author{
Călin HUSAR ${ }^{1 *}$, Mihai GROVU ${ }^{1 *}$, Cristi IRIMIA ${ }^{1 *}$, Anatole DESREVEAUX ${ }^{2 *}$, Alain BOUSCAYROL ${ }^{2 *}$, \\ Matthieu PONCHANT ${ }^{3 *}$ Pacôme MAGNIN ${ }^{3 *}$ \\ ${ }^{1}$ Siemens Industry Software SRL, Brasov, Romania \\ ${ }^{2}$ Univ. Lille, Arts et Metiers Paris Tech, Centrale Lille, HEI, EA 2697- L2EP, F-59000 Lille, France \\ ${ }^{3}$ Siemens Industry Software SAS, Lyon France \\ * PANDA, Grant Agreement 824256, https://project-panda.eu \\ Corresponding author: Calin.Husar@siemens.com
}

\begin{abstract}
The development of electric vehicles has been spectacular over the last 20 years, so the automotive industry has started to shift mass production of electrified vehicles. However, new electrified vehicles are required to face the needs of the users. Simulation is a key step for development of new vehicle. Organization tools, such as Energetic Macroscopic Representation (EMR), have therefore been developed to improve and speed-up the development of virtual electric vehicle models. The paper presents a comparison between functional and a structural representation on EV simulation under Simcenter Amesim. This paper studies the impact of the two representations on the simulation results and time. For this purpose, an EMR library for the Simcenter Amesim simulation tool has been developed.
\end{abstract}

Keywords-Representation, EMR, Electric vehicle, Simcenter Amesim.

\section{INTRODUCTION}

Today, in order to support a $40 \%$ cut of Greenhouse Gases (GHG) by 2030 expected from road transport1, Battery Electric Vehicles (BEV), Fuel Cell electric Vehicles (FCV), and Hybrid Electric Vehicles (HEV) must show shorter time-to-market compared to conventional fossil fuel-based cars [1]. In order to support this, the electrified vehicle developments are minimized through the use of the product lifecycle management (PLM) software for the automotive industry [2].

To face growing competitive pressures of the development of the electrical vehicles, dedicated software as Simcenter Amesim are used [3]. Two major categories of Software are generally used Erreur! Source du renvoi introuvable.. Functional-based simulation package are mainly made of library of functions, which are coupled to simulate the system (e.g. Matlab-Simulink(C). Structural-based simulations packages are mainly made of components, which are couple to represent the system structure (e.g. PSim (C)). The latest versions of modern simulation package include sometimes both types of library.
This paper aims to compare a functional and a structural approach for simulation of an EV using the same simulation package, Simceter Amesim. A functional library is developed in that aim based on the Energetic Macroscopic Representation (EMR) formalism [5].

\section{GRAPHICAL FORMALISM AND SOFTWARE}

\section{A. Energetic Macroscopic Representation}

EMR is a graphical description [5], which highlights the energy properties of a system. It organizes the system into interconnected basic elements (Fig. 1): source of energy (green oval), accumulation of energy (orange crossed rectangle), conversion of energy (orange square or circle), and distribution of energy (orange double square). All elements are connected according to the interaction principle: the product of the action and reaction variables leads to the power exchanged by connected elements [6]. Furthermore, all the components are described respecting the physical integral causality: the output of a storage element is an integral function of its inputs [7], [8].

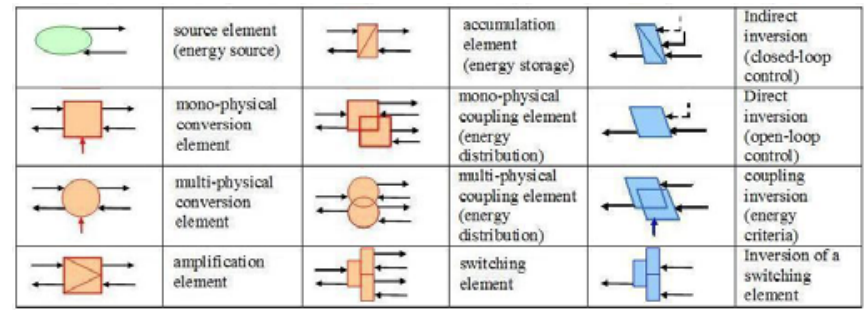

Fig. 1. Pictograms of EMR and its inversion-based control

EMR is a functional description, i.e. it focus of the subsystem function instead of the subsystem physical structure [9], [10]. In that aim, only signal variables are exchange between elements. These signal variables are inputs and outputs, which are organized according to the causality and interaction principles.

An EMR a library has been developed within the Simcenter Amesim software where all components defined by EMR 
theory are included in the library. Each EMR or control element is an empty subsystem containing only the input and output ports. Its internal structure is defined by the user, depending on what it should represent. The existing Signal and Control library from Simcenter Amesim software is used to create EMR simulation models.

\section{B. Simcenter Amesim}

The current study is performed by using the modeling and simulation capabilities of Simcenter Amesim software, an integrated, scalable system simulation platform which allows system simulation engineers to virtually assess and optimize the mechatronic systems' performance.

For power subsystems, comments libraries are used. The system is thus organized in a structural approach. Their models are linked by physical links as in the real life. For example, the connection between two electrical components is described by two wires; the connection between to rotating parts is described by a mechanical link.

The sketch of a classical Simcenter Amesim electric vehicle model is shown in fig. 2. It is composed of a longitudinal vehicle model, a quasi-static electric motor model and a quasi-static battery model.

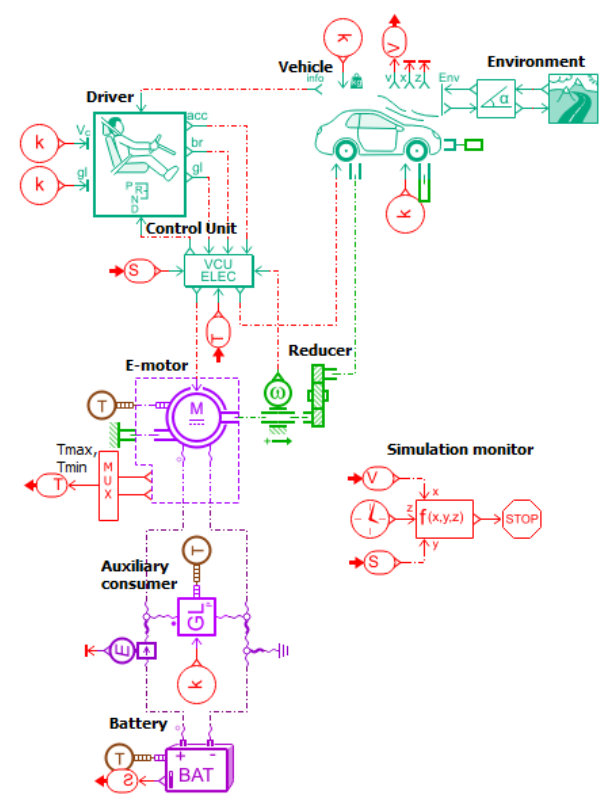

Fig 2: Sketch of a simple electric vehicle model

\section{Functional vs Structural representation}

This paper deals with the analysis and simulation of an electric vehicle, coupling functional and structural approaches in the same simulation environment.

Structural softwares are dedicated to simulate systems on the basis of their topology. Using components library, the user can easily describe the system by connecting element as in the real system. A specific solver is then used to compute in the best way equivalent relationships. Association conflicts are thus solved and sometimes forbidden by the solver.
In the functional approach, the object is described according to its function. This representation might seem more virtual, because it does not reproduce necessarily what can be seen from the system. The functionality of the system can be described using mathematical equations, transfer functions or graphic representations. Functional approaches are mainly used for analysis, abstraction or control development. As the functional approach is more general than the structural approach, the EMR graphical formalism can be applied in different software's.

In Fig. 3. a mono-physical coupling element is shown with its initial internal structure (A). It contains six ports. The modification of a mono-physical coupling to represent a differential is shown in the same figure (B). An ideal differential is modeled as two gains, for torque and rotation speed. The complexity of the model behind each of the EMR element is depending on the project requirements. Same differential is represented in (C) with a classical Simcenter Amesim element.
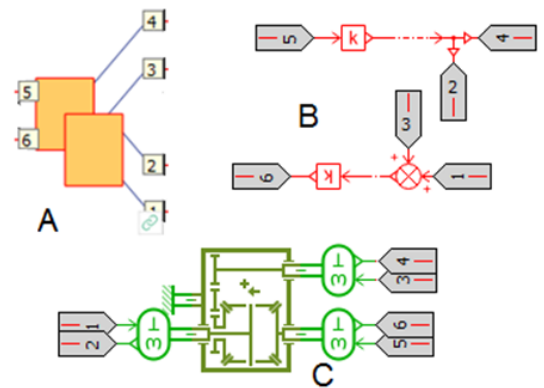

Fig. 3. Differential represented by a Mono-Physical Coupling element (A) with functional (B) and structural (C) representation

\section{Presentation of the StUdied Vehicle}

\section{A. The simulated EV}

The studied vehicle (fig.4) is driven by one electric machine. The machine technology is a permanent magnet synchronous machine. The system will be modelled via Energetic Macroscopic Representation (EMR) which as a functional approach. The control of this vehicle is applied and validate trough both cases.

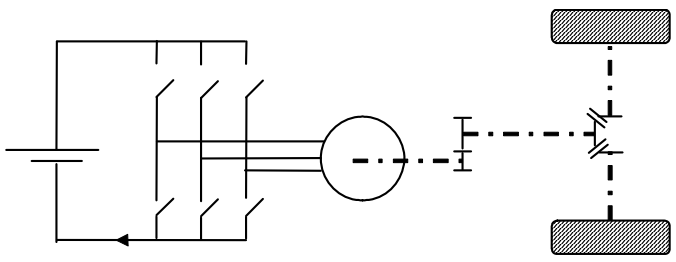

Fig. 4. Studied EV

\section{B. Subsystems specifications}

The different subsystems are defined using the modeling equations of each component.

In order to simplify the system, in this paper the battery will be modelled by a simple source of DC voltage and the PMSM will be replaced by a DC machine. The chopper is considered with a constant efficiency and the permanent magnet DC machine has the following characteristics: 
- nominal useful power : $65 \mathrm{~kW}$;

- nominal voltage : $400 \mathrm{~V}$;

- nominal current : $162 \mathrm{~A}$;

- nominal speed : $2840 \mathrm{tr} / \mathrm{min}$;

- armature resistance : $350 \mathrm{~m} \Omega$;

- armature inductance : $6.5 \mathrm{mH}$.

The mechanical transmission is composed of a gearbox with the reduction ratio 5 , a mechanical differential and one equivalent wheel with the diameter $520 \mathrm{~mm}$. The chassis can be represented with an equivalent mass (including the mass of the passengers) of $1600 \mathrm{~kg}$.

\section{STUDIED REPRESENTATIONS}

\section{A. Structural representations}

The main component used in the structural representation are: the battery, the DC machine, the gear ratio, the differential, the rotary load, the shaft with rotary spring and damper, the rotary-linear modulated transformer and the vehicle mass [11].

The battery is replaced in simulation by a variable voltage source, which is an ideal voltage sources with no dissipation.

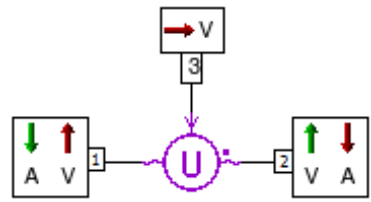

Fig. 5. The battery model

The electrical motor used is a linear DC machine. The equivalent electrical circuit of a DC machine armature in Simcenter Amesim is considered in fig. 6. The single phase electrical ports are associated on the icon with a dot. The winding connection, as well as the armature voltage and currents are defined with the convention in Fig. 6. The DC machine element from the Simcenter Amesim library contains this internal description and highlight the different ports to be connected to other elements (Fig. 7).

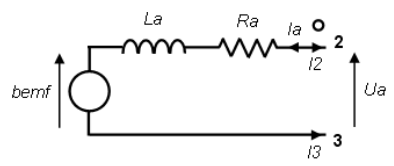

Fig. 6. Internal description of the DC machine in Simcenter Amesim

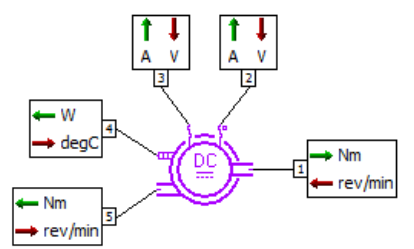

Fig. 7. External description of the DC machine in Simcenter Amesim

This description leads to the following modelling equations. The armature winding releationship connects the chopper voltage $u_{c h}$, the machine emf $e_{d c m}$ and the machine currentt $i_{d c m}$ : $u_{c h}=L \frac{d}{d t} i_{d c m}+R i_{d c m}+e_{d c m}$

The electromechanical relationships link the machine current to the machine torque $T_{d c m}$, and the rotation speed $\Omega_{\text {gear }}$ to the emf:

$$
\left\{\begin{array}{c}
T_{d c m}=k_{d c m} i_{d c m} \\
e_{d c m}=k_{d c m} \Omega_{\text {gear }}
\end{array}\right.
$$

The rotation speed is derived from the machine torque and gear torque $T_{\text {gear }}$ :

$$
T_{d c m}=J \frac{d}{d t} \Omega_{\text {gear }}+f \Omega_{\text {gear }}+T_{\text {gear }}
$$

The gearbox used in simulation is a rotary mechanical gear ratio, which multiplies the velocity in rev/min input by the gear ratio set by the user to compute the velocity output. The torque input is also multiplied by this gear ratio to compute an output torque.

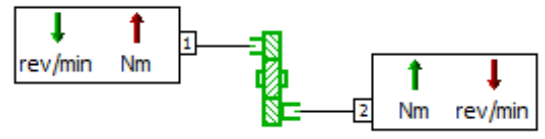

Fig. 8. The gearbox model

The classic differential used (and presented in fig. 3C) is a very simple model of differential of a car where:

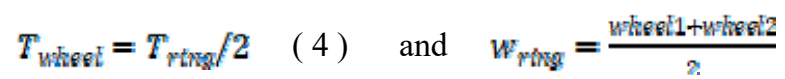

The wheels of the vehicle are replaced in by using a rotary load element, a simple dynamic model under the action of two external torques in $\mathrm{Nm}$ applied to its two ports.

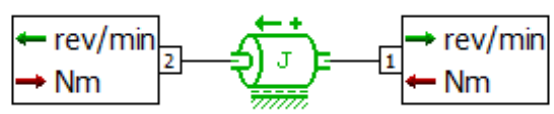

Fig. 9. Rotary load element

The rotary velocity and angle are integrated with the following equations:

$$
\text { l. } \text { accel }=I \frac{d w}{d t}=\operatorname{tar} q 1-\operatorname{tor} q 2-\text { Tvisc }
$$

The road coupling is simulated by using two different elements: a shaft connected to the rotary load and to a rotary linear modulated transformer.
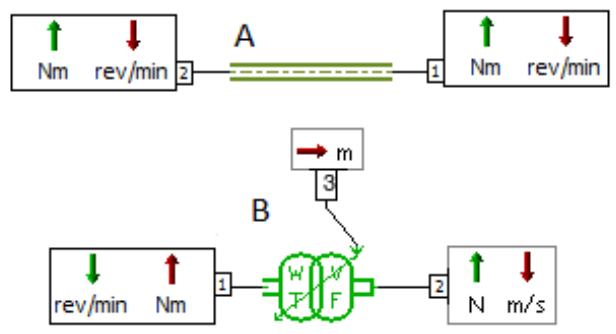

Fig. 10. The road coupling elements: the shaft (A) and the linear modulated transformer (B)

The shaft used is an elastic rotary element, acting like a rotary spring damper. The model has two ports and gives a 
torque in $\mathrm{Nm}$ as output at both these ports. Connected models must provide velocities in rev/min.

The relative velocity of the two ports is:

$$
w r o l-w 1+w 2
$$

And the relative angular displacement:

$$
a r e=\int w r e l
$$

The resulting torque is:

$$
t 1=t 2=\text { ktor:are }+ \text { rtor: } w r e l
$$

The modulated transformation between the rotary shaft and a linear shaft is done by a rotary - linear modulated transformer element. The inputs of element are the torque $t_{q}$ in $\mathrm{Nm}$ at one port and the linear velocity $v_{2}$ in $\mathrm{m} / \mathrm{s}$ at other port. The input signal $x$ at third port acts as the transformer ratio between the first two ports. The outputs are the angular velocity $w$ in $\mathrm{rev} / \mathrm{min}$ at one port and the force $f$ in $N$ at the other port.

The vehicle mass represent one-dimensional motion of a two ports mass under the action of two external forces in $N$. The model returns the velocity in $\mathrm{m} / \mathrm{s}$, the displacement in $m$ and the acceleration of the mass in $\mathrm{m} / \mathrm{s} / \mathrm{s}$.

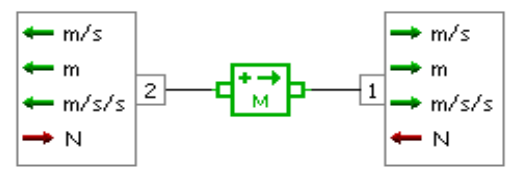

Fig. 11. The vehicle equivalent mass

The acceleration and the derivatives of the two state variables are set as follows:

$$
\begin{aligned}
& \text { accel }=\frac{d v a}{d t}=\frac{f k-f a}{n \pi a s s}+9.81 \sin (\text { thet } \alpha) \\
& \frac{d x}{d t}=v a
\end{aligned}
$$

\section{B. EMR as functional description}

The global EMR functional model is described in Fig. 11 where the upper part represent the EMR of the vehicle traction system and the lower part the control structure [12].

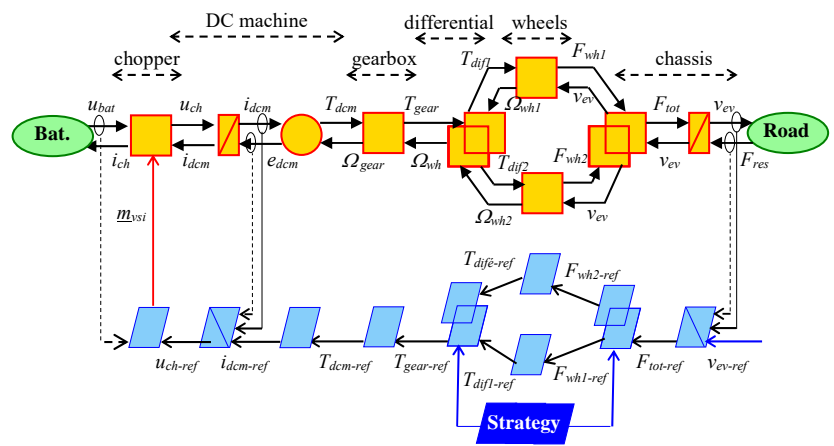

Fig. 11. EMR of the studied vehicle

The battery (oval) delivers the de voltage $v_{\text {bat }}$ and supply the traction system, which produces a current $i_{\text {tot }}$
The chopper (squares) leads to the chopper voltage $u_{\text {ch }(i)}$ from battery voltage $v_{b a t}$, and the chopper current $i_{c h(i)}$ from the machine current $i_{e m(i)}$

$\left\{\begin{array}{l}u_{c k}(t)=m(d) v_{b a t} \\ i_{\operatorname{cs}(t)}=m_{(n)} v_{e m}(t)\end{array}\right.$

with $m$ modulation of the chopper

The armature winding of DC machine, which accumulates energy (rectangles), leads to the armature current $i_{e m(i)}$ as the state variable from the e.m.f. $e_{(i)}$ and the chopper voltage $u_{c h(i)}$

$u_{c b(t)}-e_{(t)} L_{\text {irm }(t)}=\frac{d}{d t} i_{e m(t)}+R_{\text {rrm(t) }} i_{\text {em }(t)}$

where $L_{\text {arm })}$ and $R_{\text {arm })}$ are the winding inductance and resistance. The DC machine torque $T_{e m}$ is obtained from $i_{e m}$. The e.m.f. $e$ is linked to the rotation speed $\Omega_{\text {gear }}$

$f_{e m(t)}=k_{d c m(t)} t_{e m(t)}$

$\left\{e_{t h)}=\dot{k}_{\text {dsm }} \Omega_{\text {gecr }}(t)\right.$

where $k_{d c m}$ is the torque coefficient of the electromechanical conversion (circles).

The reduction gear torque $T_{\text {gear }}$ and its rotation speed $\Omega_{\text {gear }(i)}$ are obtained from the machine torque $T_{e m}$ and the rotation speed of the wheel $\Omega_{w h}$ (square):

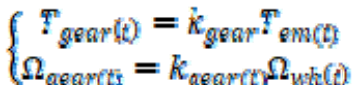

where $k_{\text {gear }}$ is the reduction gear ratio.

The wheel traction force $F_{w h(i)}$ is obtained from the gear torque $T_{\text {gear }(i)}$ and the well rotation speed from the well velocity $v_{e v}$ using the wheel radius $R_{w h}$ (square):

$\left\{\begin{array}{l}F_{\text {wh }}(t)=\frac{1}{R_{w \sigma k}} T_{e m(t)} \\ \Omega_{w F(t)}=\frac{1}{R_{w}} v\end{array}\right.$

A coupling device (double triangle) yields both the total traction force $F_{\text {tot }}$ and the wheel velocities $v_{w h(i)}$ :

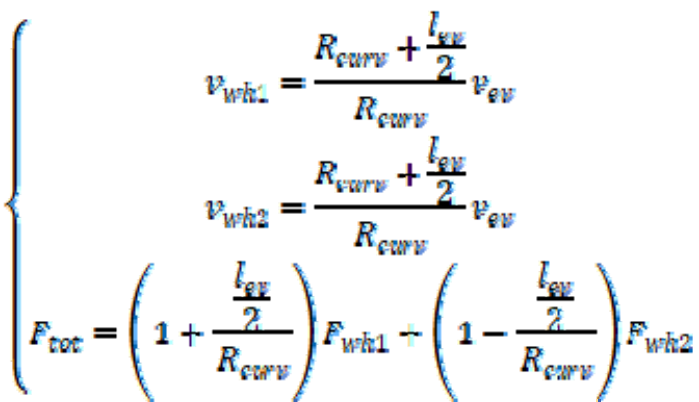

where $l_{e v}$ is the width of the vehicle and $R_{c u r v}$ is the radius of the curve defined by the steering angle. The vehicle speed $v_{e v}$ is obtained by dynamics relationships:

$M \frac{d}{d t} v_{a x}=F_{r e t}^{2}-F_{r e s}^{3}$ 
with $M$ the vehicle mass and $F_{\text {res }}$ resistant force. This relationship is represented by an accumulation element (rectangular) with the vehicle speed $v_{e v}$ as the state variable.

The environment is a mechanical source (oval) which yields the resistant force $F_{\text {res }}$

$F_{\text {ros }}=F_{0}+a v_{e v}+b v_{e v}^{2}+M G \sin \propto$

with $F_{0}$ the initial rolling force, $a$ the rolling coefficient, $b$ the drag coefficient, $\alpha$ the slope rate and $G$ the gravity.

The control of the entire system is systematically deduced from the EMR where the vehicle elements are inverted by closed-loop control.

The first inverted element is the chassis, an accumulation element where a controller is needed to define the traction force from the vehicle velocity reference where $\mathrm{C}$ is the controller.

$$
F_{\text {tot }}=c\left[v_{\text {evgef }}-v_{\text {ev_mea }}\right]+F_{\text {res mea }}
$$

Inversion of wheels it is a time-independent relationship and can be directly inverted.

\section{$T_{\text {geartety yef }}=R_{\text {wol }} F_{\text {wetothyef }}$}

Relation (15) is directly inverted to obtain the torque reference $T_{\text {em_ref }}$ from the gearbox torque reference:

$$
T_{\text {en(t)_ref }}=\frac{1}{l_{\text {gear }}} T_{\text {geartit)_yef }}
$$

The reference current $i_{\text {em(i)_ef }}$ from the DC machine inversion is deduced from the direct inversion of (14):

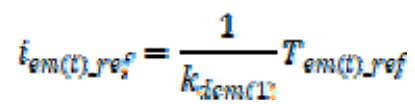

As the winding is an accumulation element (13), its inversion requires a controller:

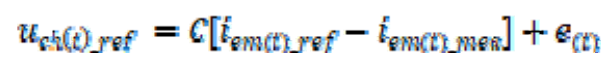

The chopper modulation $m_{\text {ch }}(i) r e f$ is deduced from the inversion of (12) with the measurement of the battery voltage vbat_mea

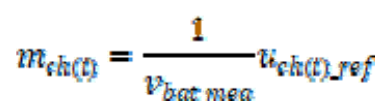

The solver of Simcenter Amesim will combine all equations in order to provide the right results.

\section{SIMULATION COMPARISON}

\section{A. Simulation organization in Amesim}

As stated previously an EMR library has been developed in Simcenter Amesim. The studied EV is thus developed using its EMR (Fig. 8).

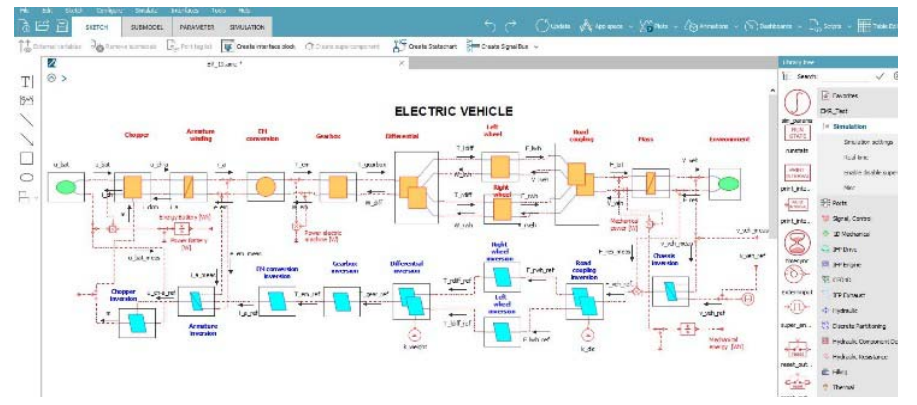

Fig. 8. EMR-based simulation of the EV in Simcenter Amesim

After a first functional model compilation four algebraic loop was displayed. The dynamical relationships of the shaft leads to the speed as state variable in conflict with the vehicle velocity. This can affect the solution produced by the solver by including algebraic loops in the model. Simcenter Amesim solver can resolve this conflict, but this is not authorized in EMR, so merging and permutation rules have to be used.

Once the algebraic loop path is known, to solve it there are two ways [13]: either by changing a component itself to another one with different type of I/O table or by changing the information flow through the introduction of new components. The reason for which the algebraic loop was signaled, by the compiler, was the general transfer function block. Simcenter Amesim first processes model compilation before block parametrization. This means that, at model compilation a general transfer function block is interpreted as a possible gain. Such a "false" generated algebraic loop is still handled by Simcenter Amesim. The solution found is to use an integrator block, instead of the general transfer function, which eliminates the algebraic loop.

The same vehicle is also organized using the structural library in Simcenter Amesim (Fig. 9). In order to have a fair comparison the same control organization (derived from EMR) is used.

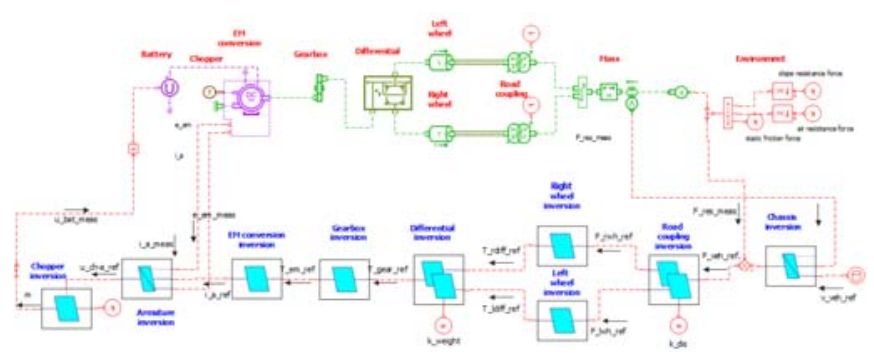

Fig.9. Structural-based simulation of the EV in Simcenter Amesim

\section{B. Simulation results}

The urban part of NEDC driving cycle is considered. For both simulation, the vehicle velocity is well achieved (Fig.10). 


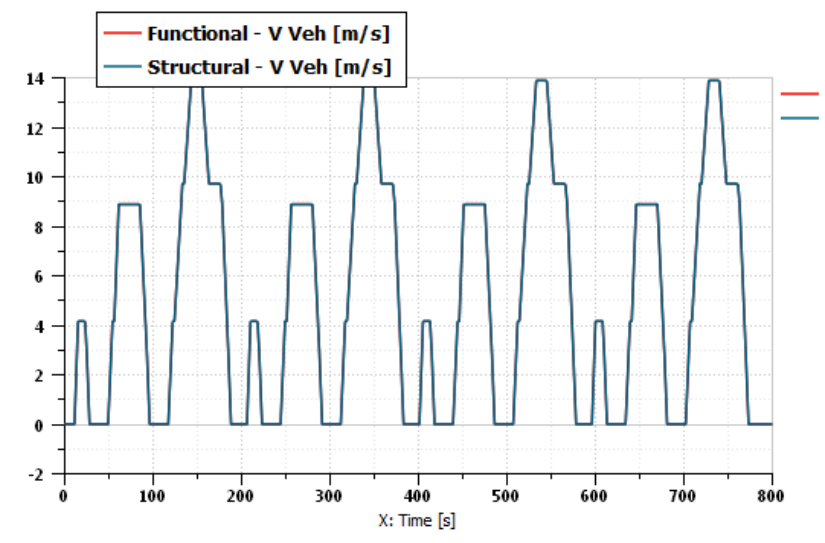

Fig. 10. Vehicle velocity

The relationships between the motor's electrical characteristics and mechanical performance are plotted for functional (Fig. 11.a) and structural (Fig. 11.b) models, where the results are almost identical.

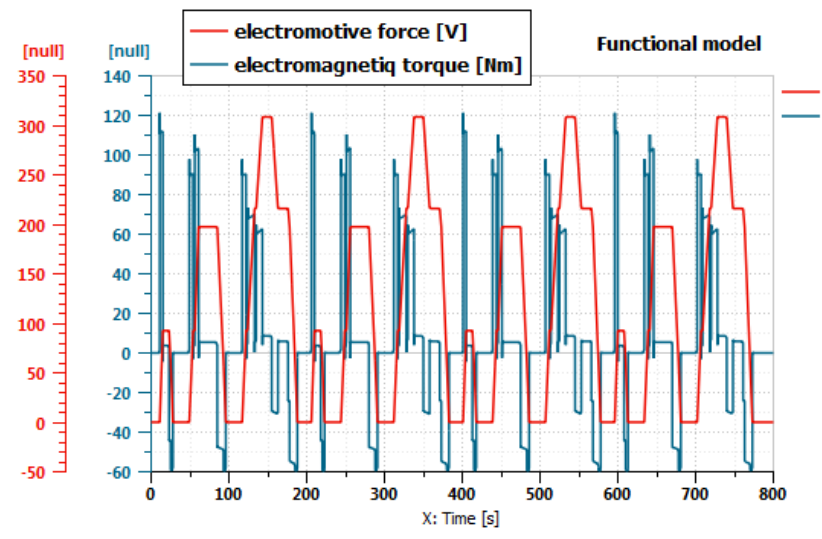

Fig. 11.a. Simulation results, electrical vs mechanical performance for functional model

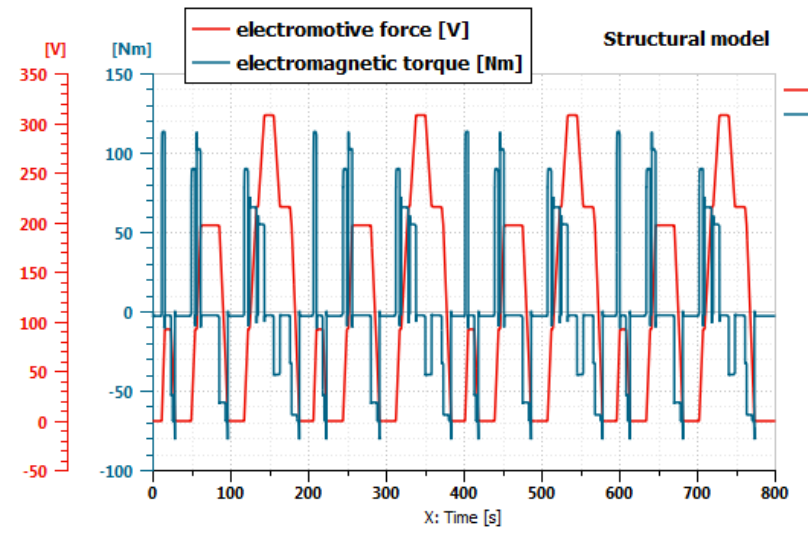

Fig. 11.b. Simulation results, electrical vs mechanical performance for structural model

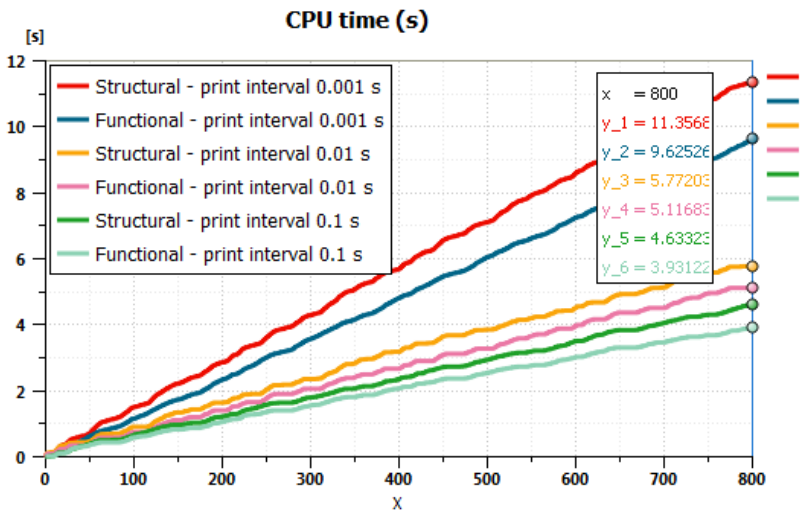

Fig 12: Simulated time (800 s) vs computation CPU time

In terms of computation time, the real CPU time of the simulation process is used to evaluate the performance of the simulation. Different print interval steps are used $(0.1 \mathrm{~s}, 0.01 \mathrm{~s}$, $0.001 \mathrm{~s}$ ) for both, structural and functional approach. After performing the simulations, we observe that for a simulated time of 800 seconds the functional simulation has about $15 \%$ lower CPU-simulation time. For the 0.01 seconds print interval, at which the results are saved in the results file, the maximal difference is 0.6 seconds and for 0.001 print interval the difference is 1.7 seconds. Under the same simulation condition, the simulation CPU time for the structural model, but without the control organization derived from EMR, is lower for all print interval cases.

\section{CONCLUSION}

Simulation is a key tool for the development of innovative electrified vehicles. In this paper the classical structural approach is composed with a pre-functional approach thanks to EMR. In that aims, an EMR library has been developed in Simcenter Amesim. The same EV has been simulated using both approaches with the same control using the same software. In terms of accuracy both approaches lead to the same results. In terms of computation time, the difference between the two different cases, is about $15 \%$. If in this simple case, it can be neglected, for more complex case, it could be to be considered.

This paper shows the main advantages of Simcenter Amesim software: an 1D simulation platform for physical modeling (structural) with block diagram (functional) capabilities.

\section{ACKNOWLEDGMENT}

This project has received funding from the European Union's Horizon 2020 research and innovation programme under grant agreement No 824256 (PANDA).

\section{REFERENCES}

[1] "Global EV outlook 2018, towards cross-modal electrification", International Energy Agency report, 2018

[2] D. W. Gao, C. Mi, and A. Emadi, "Modeling and simulation of electric and hybrid vehicles", Proceeding of the. IEEE, vol. 95, no. 4, pp. 729745, April 2007

[3] Simcenter AMESim https://www.plm.automation.siemens.com/ 
[4] C. C. Chan, A. Bouscayrol, K. Chen, "Electric, Hybrid and Fuel Cell Vehicles: Architectures and Modeling", IEEE transactions on Vehicular Technology, vol. 59, no. 2, pp. 589-598, February 2010

[5] A. Bouscayrol, J.-P. Hautier, and B. Lemaire-Semail, "Graphic formalism for the control of multi-physiscal energetic systems: COG and EMR," in Systemic Design Methodologies for Electrical Energy Systems, New York, NY, USA, Wiley, ch. 32012.

[6] A. Bouscayrol, B. Davat, B. de Fornel, B. François, J. P. Hautier, F. Meibody-Tabar, M. Pietrzak-David, "Multimachine Multiconverter System: application for electromechanical drives", European Physics Journal - Applied Physics, vol. 10, no. 2, pp. 131-147, May 2000.

[7] J.P. Hautier, P.J. Barre, "The causal ordering graph - A tool for modeling and control law synthesis", Studies in Informatics and Control Journal, vol. 13, no. 4, pp. 265-283, 2004.

[8] EMR. [Online], available: http://www.emrwebsite.org/

[9] P.J. Gawthrop, G.P, Bevn G.P., "'Bong Graph modeling, a tutorial introduction for control engineers", IEEE Control Systems magazine vol. 27, no. 2, pp. 24-45, 2007.

[10] A. Bouscayrol, P. Delarue, W. Lhomme, and B. Lemaire-Semail, "Teaching drive control using Energetic Macroscopic RepresentationFrom maximal to practical control schemes," EPE'15 ECCE-Europe, Geneva, Switzerland, Sep. 8-10, 2015.

[11] Simcenter Amesim, Help

[12] K.Chen, A. Bouscayrol and W. Lhomme, "Energetic Macroscopic Representation and Inversion-based Control: Application to an Electric Vehicle with an Electrical Differential", Journal of Assian Electric Vehicles, Volume 6, Number 1, June 2008

[13] Simcenter Amesim, Platform Documentation, Solvers and Numerics, 4 Algebraic loops within Simcenter Amesim 